\title{
A Case Report of the Symptom-Relieving Action of an Anterior Flat Plane Bite Plate for Temporomandibular Disorder
}

\author{
Kengo Torii ${ }^{*}, 1$ and Ichiro Chiwata ${ }^{2}$ \\ ${ }^{1}$ Torii Dental Clinic, 1-23-2 Ando, Aoi-ku, Shizuoka-shi, 420-0882 Japan \\ ${ }^{2}$ Chiwata Dental Clinic, 2-1-3 Gofuku-cho, Aoi-ku, Shizuoka-shi, 420-0031 Japan
}

\begin{abstract}
Splint therapy is a widely used modality for temporomandibular disorders (TMDs). However, questions remain regarding the mechanism of symptom relief. Recently, a relation between TMD and a discrepancy between the habitual occlusal position (HOP) and the anterior flat plane bite plate-induced occlusal position (BPOP) has been reported. Therefore, to understand the mechanism of the symptom relief associated with the use of a bite plate, the changes in both the HOP and BPOP values were observed during anterior bite plate treatment in a patient with TMD.
\end{abstract}

\section{Case presentation}

Anterior bite plate treatment was started in a patient complaining of arthralgia. A discrepancy between the HOP and BPOP values was recorded three days after the first visit; this discrepancy disappeared on day 6 , and the disappearance was maintained between days 6 and 12. The disappearance was associated with a decrease in the severity of the patient's symptoms; however, the discrepancy reappeared after 15 days, coinciding with the complete disappearance of the patient's symptoms. Therefore, the effect of the bite plate on the resolution of the discrepancy between the HOP and BPOP values was interpreted as being unsustainable and occlusal equilibration for the BPOP was performed at 17 days. Thereafter, neither a discrepancy in the HOP and BPOP values nor any symptoms of TMD (right temporomandibular joint pain or limited jaw opening) reoccurred during a two-year follow-up period.

\section{Conclusion}

Within the limitations of the present study, the symptom-relieving action of an anterior flat plane bite plate on the resolution of occlusal discrepancy appeared to be temporary. This outcome supports previously reported conclusions that the effectiveness of stabilization splint therapy for reducing symptoms in patients with pain dysfunction syndrome does not differ significantly from that of other conservative therapies or of no treatment at all.

Keywords: Anterior flat plane bite plate, bite plate-induced occlusal position, occlusal discrepancy.

\section{INTRODUCTION}

Occlusal splints are widely used for the treatment of temporomandibular disorders (TMD), even though their mechanism of action is unknown. In particular, an anterior flat plane bite plate has been used as a provisional appliance for decreasing the painful symptoms [1], since such bite plates are easy to fabricate and to adjust. Regarding the mechanism of their action, the electromyographic activities (EMG) of patients using various splints have been reported [2-6], and mandibular shifts after splint use have been reported in some cases [7]. However, the mechanism continues to be uncertain. The clinical effects of such splints in patients with TMD, particularly the effects on myofascial pain and the effects on temporomandibular joint (TMJ), have also been reported and remain controversial [8-14]. In a recent study, a relation between the symptoms of TMD and a discrepancy between the habitual occlusal position (HOP) (voluntarily closed position while the subject is in an upright position)

*Address correspondence to this author at the Torii Dental Clinic, 1-23-2 Ando, Aoi-ku, Shizuoka-shi, 420-0882 Japan; Tel: 054247 2966; Fax: 054 248 2130; E-mail: wbs89508@ mail.wbs.ne.jp;wbs55314@ mail.wbs.ne.jp and the bite plate-induced occlusal position (BPOP) (voluntarily closed position while the subject is in an upright position after an anterior flat plane bite plate has been worn for a few minutes and then removed) was reported [15]. Good treatment outcomes have been obtained by eliminating such occlusal discrepancy through occlusal equilibration of the BPOP [16]. Therefore, the present study aimed to understand the mechanism of symptom-relief by the use of an anterior flat plane bite plate by observing changes in the HOP and BPOP values and comparing them with changes in the severity of symptoms.

\section{CASE PRESENTATION}

A 61-year-old man presented with a one-week history of pain in the right TMJ area, especially during eating, and a restricted range of jaw motion. The patient's previous medi$\mathrm{cal} /$ dental history was unremarkable. No history of parafunction was present, and the patient had not experienced any TMJ pain prior to the presently reported experience. An examination revealed no deviation in the opening path and no noise in the TMJ.

The maximum unassisted opening distance was $40 \mathrm{~mm}$. The patient reported pain upon palpation of the right TMJ 


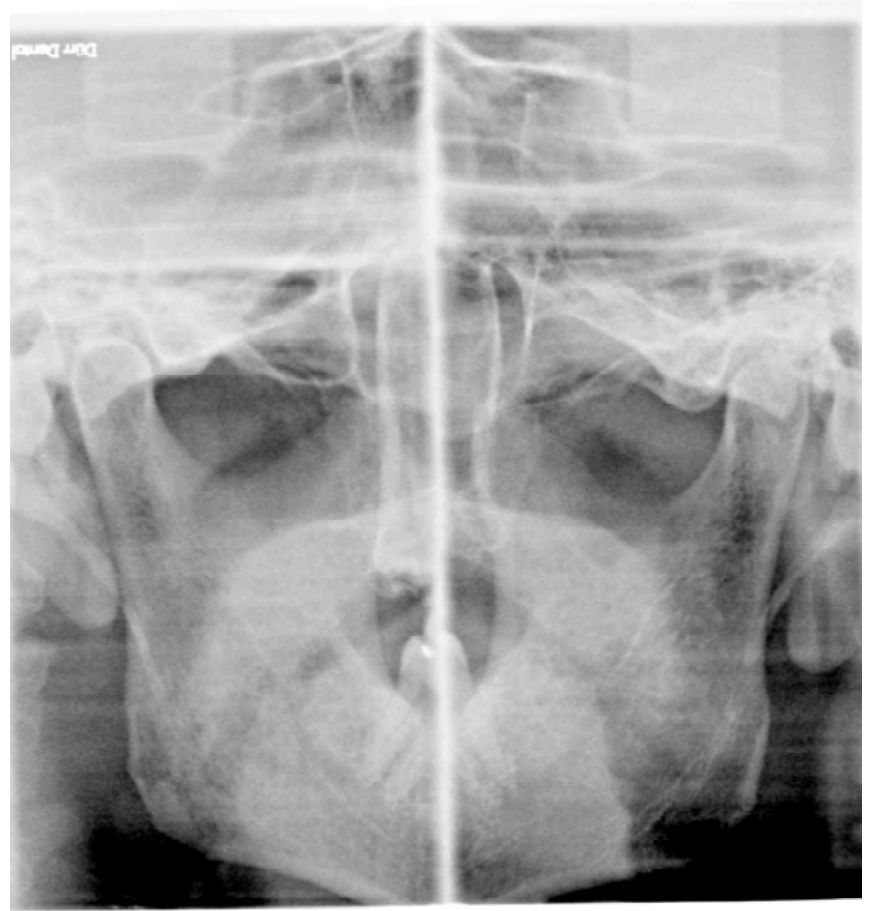

Fig. (1). Tomograph of the patient. The right TMJ shows no evidence of degeneration.

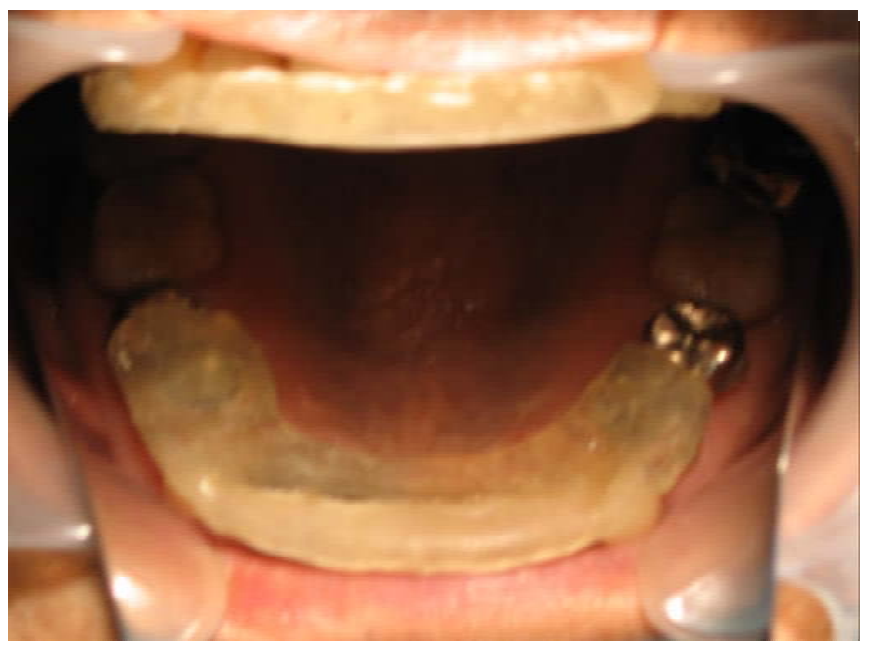

Fig. (2). Anterior flat plane bite plate. The plate covers the six upper anterior teeth and the first premolar teeth on both sides.

(lateral pole, inside ear), but had no extraoral or intraoral muscle tenderness. Twenty-eight teeth were present, and the occlusion was anatomically normal. TMJ tomography showed no evidence of degeneration (Fig. 1), and a diagnosis of temporomandibular arthralgia was made based on the Research Diagnostic Criteria for TMD (RDC/TMD) [17]. Since an anterior flat plane bite plate has been recommended as a provisional appliance to decrease painful symptoms [1], an anterior flat plane bite plate covering the upper six anterior teeth and the first premolar teeth on both sides was fabricated for short term use using self-curing acrylic resin (GC, Tokyo, Japan). The occlusal surface of the plate was made flat and perpendicular to the mandibular incisors to allow free movement in all directions (Fig. 2). Medication was not prescribed.

The patient was instructed to wear the plate during the day, except when eating or speaking. This appliance therapy was continued until the symptoms were alleviated; meanwhile, the HOP and BPOP were measured as follows. The HOP (first) and BPOP (second) were recorded over time. The HOP, obtained by voluntary jaw closing while the patient was seated in an upright position with the occlusal plane parallel to the floor, was assumed to be the mandibular position imposed by the motor program of the central nervous system, and was defined as the stable (intercuspal) position. The BPOP, attained during voluntary jaw closing while the patient was seated in an upright position after the patient had worn an anterior flat plane bite plate for five minutes, was assumed to be the muscular position achieved by altering or deprogramming the motor program. This procedure to obtain the muscular position was established in a previous study [15]. To record the HOP, a vinyl polysiloxane bite registration material (GC, Tokyo, Japan) was applied over the occlusal surfaces with a syringe and the patient was asked to swallow and then close his mouth to maximum intercuspation, then hold that position until the material set (approximately one minute). To standardize the BPOP recording method, the patient was conditioned neuromuscularly using an anterior flat-plane bite plate, against which the patient tapped and slid his anterior lower teeth for a period of five minutes. After conditioning, the bite plate was removed and the registration material was applied over the occlusal surface; the patient was then asked to close his mouth until the upper and lower teeth just came into contact with each other and to hold that position. Three inter-occlusal recordings were made for each of the occlusal positions at each visit. A dentist who was not involved in the recording and who was unaware of the patient's status performed the following measurements and analysis. The trimmed intraoral records were interposed between the cast on the threedimensional apparatus of a modified articulator (Fig. 3), and occlusal papers of different colors were interposed between the recording surfaces and the recording needles, marking the positions. All registrations were read under a measuring microscope (Pika Seiko, Tokyo Japan) with a resolution of $0.01 \mathrm{~mm}$. The statistical significance of the differences between the HOP and BPOP measurements recorded on each day and between two HOP and two BPOP recordings made on different days were calculated using an analysis of variance (ANOVA) for a two-factor experiment with repeated measurements for both position factors. The statistical significance of the differences in the variations of the two positions recorded on two different days was also calculated using an ANOVA. Significance levels of $p<0.05, p<0.01$, and p0.005 were established. In addition, the maximum unassisted opening and the pain score on a 10-point Visual Analogue Scale (VAS), where 0 denoted "no pain" and 10 denoted "worst pain" were recorded over time. The changes in the symptoms, the discrepancy between the HOP and BPOP values, and the variations in the HOP and BPOP values are shown in Figs. 4 and 5. On day one, the difference between the HOP and the BPOP was not statistically significant (Fig. 4). On day 3, however, the difference between the HOP and the BPOP at this time-point was significant $(\mathrm{p}<0.005)$. The 


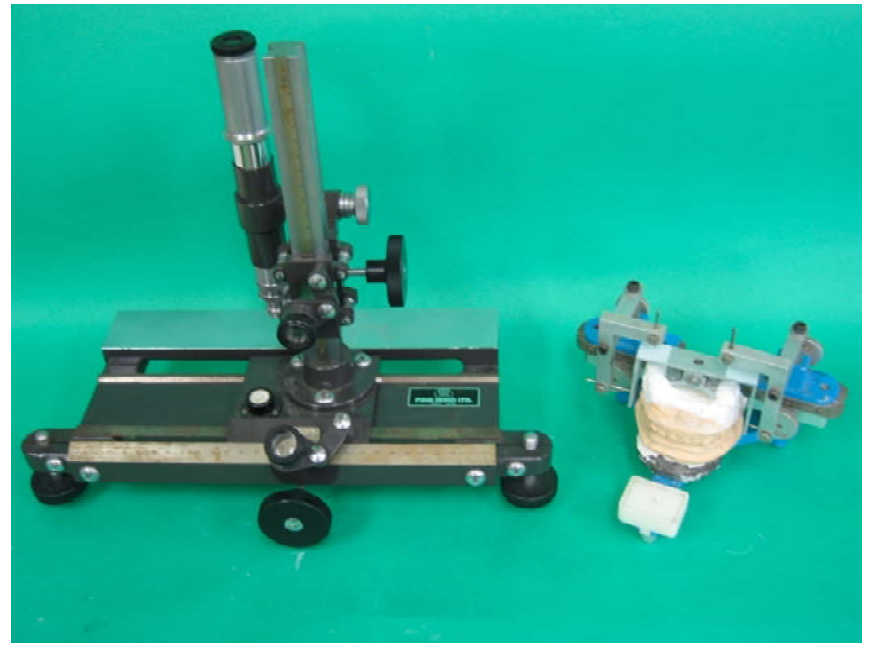

Fig. (3). Mandibular position analyzer and measuring microscope. The apparatus added to an articulatorfor the three-dimensional analysis of the mandibular position consists of right and left recording arms with pins in the condylar post holes. The recording frame is attached to the upper cast. The mandibular positions are recorded on the frame by the pins on both sides.

difference between $B_{1}$ and $B_{2}$ was also significant $(p<0.05)$, while that between $\mathrm{H}_{1}$ and $\mathrm{H}_{2}$ was not $(\mathrm{p}>0.1)$. The mean differences between the HOP and BPOP were $0.18 \pm 0.14$ $\mathrm{mm}$ (mediolateral), $0.71 \pm 0.68 \mathrm{~mm}$ (anteroposterior) and $0.45 \pm 0.34 \mathrm{~mm}$ (superoinferior). On day 15, the patient's maximum unassisted opening increased to $45 \mathrm{~mm}$, the VAS score remained zero, and the difference between $\mathrm{H}_{6}$ and $\mathrm{B}_{6}$ was significant $(p<0.01)$, while that between $B_{5}$ and $B_{6}$ was not significant $(\mathrm{p}>0.1)$ (Fig. 4). If this discrepancy had been left untreated, the symptoms would have recurred. Therefore, for ethical reasons, occlusal equilibration was performed for the BPOP after obtaining the patient's informed consent. The discrepancy between the HOP and BPOP values was significant on day 25 , and the patient reported cheek biting on his left side. However, the discrepancy disappeared by day 33, and the cheek biting ceased. The difference in the BPOP between the visits on day 3 and day 110 was not significant ( $>0.1)$. The variations in the BPOP values measured on days $25,33,50$ and 110 were significantly smaller than those measured on day 15 ( $\mathrm{p}<0.01)$ (Fig. 5). The HOP shifted anterolaterally to the left after occlusal adjustment. The patient's symptoms (right TMJ pain while eating and limited jaw opening) did not recur during a two-year follow-up period.

\section{DISCUSSION}

Manns et al. [2] recorded the electromyographic (EMG) activities for various vertical occlusal dimensions and obtained minimal basal EMG activities at distances of $10 \mathrm{~mm}$ $16 \mathrm{~mm}$ away from the occluded position. These results possibly support the hypothesis that wearing an occlusal splint induces muscular relaxation, reducing myospasms and resulting in a decrease in EMG activity in the area of the jaw muscles. In addition, Manns et al. [3] reported that elevator muscular activity during the use of an anterior block (like as anterior bite plate) was significantly lower than that during the use of three other posterior blocks and lower than that for an interocclusal position. In the present study, muscular relaxation seemed to have occurred with the wearing of an anterior flat plane bite plate from the first visit until day 3, and this phenomenon might have been induced by a reduction in elevator muscle activity as a result of anterior bite raising $[2,3]$. Consequently, the BPOP, which presents a physiological muscular position, was isolated from the combined HOP-BPOP position as a result of the muscular relaxation. The disappearance of the occlusal discrepancy between the HOP and the BPOP between day 6 and day 12 seemed to have been induced by the reprogramming of the voluntary

\begin{tabular}{|c|c|c|c|c|c|c|c|c|c|c|}
\hline NS & H1 B1 & $\mathrm{H}_{2}$ & & & & $\mathrm{H}_{6}$ & $\mathrm{H}_{7}$ & & & \\
\hline $\begin{array}{l}\uparrow \\
S \\
\downarrow\end{array}$ & & & & & & & & & & \\
\hline NS & & $\mathrm{B}_{2}$ & H3 В3 & H4 B4 & H5 B5 & $\mathrm{B}_{6}$ & B7 & $\mathrm{H}_{8} \mathrm{~B}_{8}$ & $\mathrm{H}_{9} \mathrm{~B}_{9}$ & $\mathrm{H}_{10} \mathrm{~B}_{10}$ \\
\hline Days & 1 & 3 & 6 & 9 & 12 & 15 & 25 & 33 & 50 & 110 \\
\hline VAS & 6 & 3 & 2 & 2 & 0 & 0 & 0 & 0 & 0 & 0 \\
\hline Max. & 40 & 42 & 42 & 42 & 42 & 45 & 45 & 45 & 45 & 45 \\
\hline
\end{tabular}

Occlusal adjustment was performed on day 17 ,

NS: Not significant

S: Significant

Days: Days of visits

$\mathrm{H}_{1-10}$ : Habitual occlusal position on different days

$\mathrm{B}_{1-10}$ : Bite plate-induced occlusal position on different days

VAS: Scores on a 10-point Visual Analogue Scale

Max.: Maximum Unassisted Opening (mm)

Fig. (4). HOP and BPOP positions and TMD symptoms on different days. 


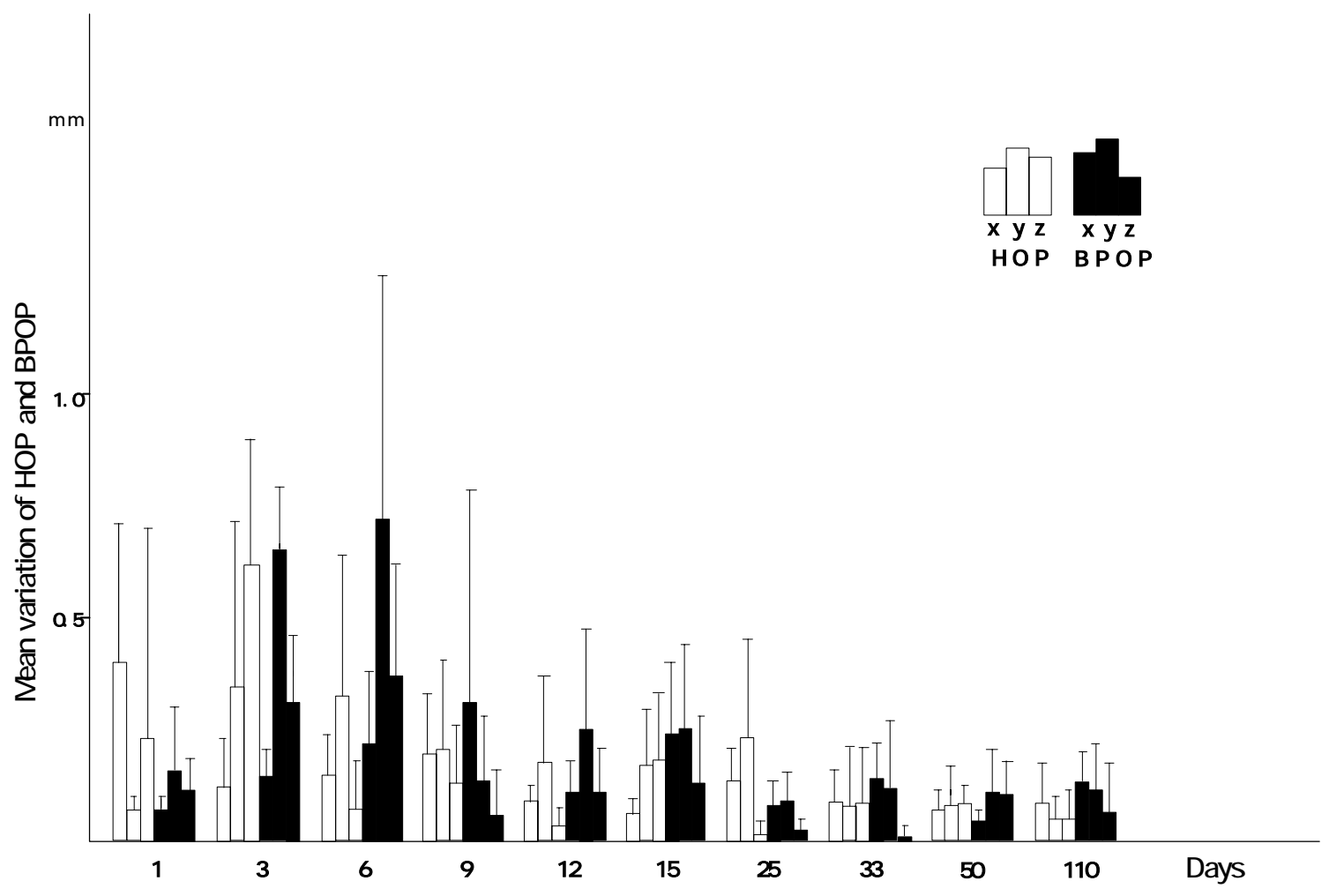

Fig. (5). Mean variations in the three axes on different days. x: mediolateral; y: anteroposterior; z: superoinferior. HOP: habitual occlusal position; BPOP: bite plate-induced occlusal position.

jaw closing motor program arising from the central nervous system having learned a mandible position that reduces pain, and mandible closure at the BPOP shifted from the previous HOP. The existence of an occlusal discrepancy between the HOP and the BPOP means that when the mandible voluntarily closes, the elevator muscles require additional activity to adapt the mandible from the BPOP to the HOP as multiple teeth come in contact with a stable position during the change from isotonic muscle contraction to isometric. This condition may cause muscle fatigue, resulting in muscle pain. The elimination of such a discrepancy through occlusal equilibration reduces the activity of the muscles and consequently reduces and alleviates the painful symptoms of TMD, as reported in the study by Torii and Chiwata [16]. Therefore, the reduction in the severity of the symptoms between day 6 and day 12 was thought to have been caused by the disappearance of the occlusal discrepancy between the HOP and the BPOP. The shift in the mandible from the previous HOP to the BPOP in the present study was comparable to the results of a study by $\mathrm{Fu}$ et al. [7]. Fu et al. [7] reported that after flat bite plate therapy, the mandibular position of all TMD patients shifted by about $1.16 \mathrm{~mm}$ toward the labial frenum midline position. The value of this shift was larger than that observed in the present study. The shift in the present study was measured at the first molar. On the other hand, the existence of the occlusal discrepancy between the HOP and the BPOP seems to distort the normal relationship between the disc and the condyle in the TMJ, because the correct relationship between the disc and the condyle is thought to be fundamentally established during the development of the functions of the masticatory muscles (with re- gard to the muscular position, that is the BPOP) before tooth eruption. Although Ekberg et al. [10] reported the effects of a stabilization splint on the condylar position using radiography, the slight shift (less than $1 \mathrm{~mm}$ ) in the present study would have likely been difficult to detect using radiography. The reappearance of the occlusal discrepancy between the HOP and the BPOP on day 15 in the present study might be interpreted as supporting the hypothesis that the simultaneous isometric contraction of the elevator muscles requires a stable mandibular position involving multiple teeth contacts under normal conditions (absence of pain) and, consequently, the mandible returned to its previous stable position once the symptoms had disappeared. Thus, the discrepancy between the HOP and the BPOP persisted, and if the discrepancy had been left untreated, the symptoms would have recurred after the cessation of bite plate wearing, similar to the outcome reported by Sheikholeslam and Riise [13]. Therefore, the effect of occlusal splints on TMD symptoms seems to be only temporary, lasting until the symptoms disappear, and the symptoms are likely to appear intermittently during splint therapy. These conditions are likely responsible for the varying of effects of splint therapy, compared with other conservative therapies, as reported by other researchers [9,11-13]. The elimination of the occlusal discrepancy between the HOP and the BPOP is essential for the alleviation of TMD symptoms.

\section{CONCLUSION}

Within the limitations of the present study, the patient's TMD symptoms completely disappeared after the use of an anterior flat plane bite plate. However, the effect of the plate 
on the resolution of occlusal discrepancy appeared to be temporary. Therefore, if the patient had not worn the bite plate, the symptoms would have likely recurred. This outcome supports previously reported conclusions that the effectiveness of stabilization splint therapy for reducing symptoms in patients with pain dysfunction syndrome does not differ significantly from that of other conservative therapies or of no treatment at all.

\section{ACKNOWLEDGEMENT}

Written consent was obtained from the patient prior to the publication of this study.

\section{REFERENCES}

[1] McNeil C, Ed. Craniomandibular disorders. guidelines for evaluation, diagnosis, and management. Chicago: Quintessence Publishing 1990.

[2] Manns A, Miralles R, Guerrero F. The changes in electrical activity of the postural muscles of the mandible upon varying the vertical dimension. J Prosthet Dent 1981; 45: 438-45.

[3] Manns A, Rocabado M, Cadenasso P, Miralles R, Cumsille MA. The immediate effect of the variation of anteroposterior laterotrusive contact on the elevator EMG activity. J Craniomandib Pract 1993; 11: 184-91.

[4] Williamson EH, Lundquist DO. Anterior guidance: its effect on electromyographic activity of the temporal and masseter muscles. J Prosthet Dent 1983; 49: 816-23.

[5] Clark GT, Beemsterboer PL, Solberg WK, Rugh JD. Nocturnal electromyographic evaluation of myofascial pain dysfunction in patients undergoing occlusal splint therapy. J Am Dent Assoc 1979; 99: 607-11.

[6] Carr AB, Christensen LV, Donegan SJ, Ziebert GJ. Postural contractile activities of human jaw muscles following use of an occlusal splint. J Oral Rehabil 1991; 18:185-91.

[7] Fu AS, Mehta MS, Forgione AG, Al-Badawi EA, Zawawi KH. Maxillomandibular relationship in TMD patients before and after short-term flat plane bite plate therapy. J Craniomandib Pract 2003; 21: 172-9.

[8] Baldissara S, Mascellani SC, Catapano S, Badissara P. Short-term effects of the Michigan splint on muscular and joint pain. Minerva Stomatol 1998; 47: 235-8.

[9] Ekberg EC, Vallon D, Nilner M. Occlusal appliance therapy in patients with temporomandibular disorders. A double blind controlled study in a short-term perspective. Acta Odontol Scand 1998; 56: $122-8$

[10] Ekberg EC, Sabet ME, Petersson A, Nilner M. Occlusal appliance therapy in a short-term perspective in patients with temporomandibular disorders correlated to condyle position. Int J Prosthodont 1998; 11: 263-8.

[11] Ekberg EC, Vallon D, Nilner M. The efficacy of appliance therapy in patients with temporomandibular disorders of mainly myogenous origin. A randomized, controlled, short-term trial. J Orofac Pain 2003; 17: 133-9.

[12] Dao TT, Lavigne GJ, Charbonneau A, Feine JS, Lund JP. The efficacy of oral splints in treatment of myofascial pain of the jaw muscles: a controlled clinical trial. Pain 1994; 56: 85-94.

[13] Al-Ani MZ,Davies SJ, Gray RJ, Sloan P, Glenny Am. Stabilization splint therapy for temporomandibular pain dysfunction syndrome. Cochrane Database Syst Rev 2004; Issue 1. Art. NO: CD 002778.

[14] Sheikholeslam A, Holmgren K, Riise C. A clinical and electromyographic study of long-term effects of an occlusal splint on the temporal and masseter muscles in patients with functional disorders and nocturnal bruxism. J Oral Rehabil 1986; 13(2): 137-45.

[15] Torii K, Chiwata I. Relationship between habitual occlusal position and flat bite plane induced occlusal position in volunteers with and without temporomandibular joint sounds. J Craniomandib Pract 2005; 23: 16-21.

[16] Torii K, Chiwata I. Occlusal adjustment using the bite plateinduced occlusal position as a reference position for temporomandibular disorders: a pilot study. Head Face Med 2010; 6: 5.

[17] Dworkin SF, LeResche L. Research diagnostic criteria for temporomandibular disorders. Review, criteria, examinations and specifications, critique. J Craniomandib Disord Facial Oral Pain 1992; 6: 301-55.

(C) Torii and Chiwata; Licensee Bentham Open.

This is an open access article licensed under the terms of the Creative Commons Attribution Non-Commercial License (http://creativecommons.org/licenses/by-nc/3.0/) which permits unrestricted, non-commercial use, distribution and reproduction in any medium, provided the work is properly cited. 